\title{
Higher Neutrophil-to-lymphocyte Ratio is Association with an Increased Risk of Fatal Stroke Occurrence in Older Chinese
}

\section{Zhi-bing Hu}

Guangzhou Twelfth People's Hospital

\section{Ze-xiong Lu}

Sanya Central Hospital

Feng Zhu ( $\square$ chifengzhu@hotmail.com )

Guangzhou Twelfth People's Hospital

\section{Research Article}

Keywords: Neutrophil, Lymphocyte, Stroke, Ischaemic, Haemorrhagic

Posted Date: August 30th, 2021

DOI: https://doi.org/10.21203/rs.3.rs-800401/v1

License: @ (i) This work is licensed under a Creative Commons Attribution 4.0 International License. Read Full License 


\section{Abstract}

Background: The neutrophil-to-lymphocyte ratio (NLR) has linked to a mortality risk of coronary heart disease. However, it is unclear whether the NLR is related to the risk of fatal stroke in a relatively healthy elderly population.

Aims: To evaluate the association between the NLR and the risk of fatal stroke in elderly populations.

Methods: In total, 27811 participants without a stroke history at baseline were included and followed up for a mean of 11.5 (standard deviation=2.3) years. After review of available records, 503 stroke deaths (ischaemic 227, haemorrhagic 172 and unclassified 104) were recorded. Cox proportional hazards regression was used to assess the relationship between the NLR and future risk of fatal stroke, fatal ischaemic stroke or fatal haemorrhagic stroke.

Results: (1) Compared with those in the $1^{\text {st }}$ quartile and after adjustments for a series of factors, those in the highest neutrophil (NEUT) quartile had a $45 \%$ and a $65 \%$ increased risk of fatal stroke (adjusted hazard ratio (aHR) $=1.45,95 \%$ confidence interval $(\mathrm{Cl}) 1.10-1.89, P=0.008)$ and fatal ischaemic stroke (aHR=1.65, 95\% $\mathrm{Cl} 1.10-2.47, P=0.02)$, respectively; while no significant relationship was obtained between the lymphocyte (LYM) and the risk of fatal stroke. (2) The restricted cubic splines showed an increased trend of relationship between the NLR and the risk of fatal stroke occurrence. Compared with those in the lowest quartile $(\leq 1.39)$, the participants with the highest NLR $(\geq 2.24)$ had a $76 \%$ and a $115 \%$ increased risk of fatal stroke (aHR=1.76, 95\% $\mathrm{Cl} 1.33-2.32)$ and fatal ischaemic stroke $(\mathrm{aHR}=2.15,95 \%$ Cl 1.41-3.28), respectively; Similar associations for stroke and ischaemic stroke were obtained after further adjustment for C-reactive protein. (3) Compared with those in NLR $\leq 1.75$, the participants in NLR $>1.75$ had a $57 \%$ and a $91 \%$ increased risk of fatal stroke $(\mathrm{aHR}=1.57,95 \% \mathrm{Cl} 1.20-2.06)$ and fatal ischaemic stroke $(\mathrm{aHR}=1.91,95 \% \mathrm{Cl} 1.27-2.88)$, respectively. (3) As a continuous variable, the NLR presented an increased risk of fatal stroke (aHR=1.11 95\% Cl 1.06-1.17) and fatal ischaemic stroke (aHR=1.15 95\% Cl 1.09-1.21), respectively.

Conclusions: Higher NLR was associated with an increased risk of fatal stroke and fatal ischaemic stroke occurrence in a relatively healthy elderly population.The clinicians should pay more attention to asymptomatic inflammatory characteristics in relatively healthy older population.

\section{Background}

Stroke has become the second and the leading cause of deaths worldwide, 2015 [1] and in China, 2013 [2]. It is classified mainly as ischaemic and haemorrhagic stroke. Hypertension, diabete, and smoking have been known as the shared risk factors of stroke incidence, and related closely to a chronic inflammation [3-6]. Chronic inflammatory diseases were associated with an increased risk of stroke [7-9], suggesting that inflammation runs through the pathophysiological progress in stroke.

The neutrophil-to-lymphocyte ratio (NLR) has been widely used to predict various causes of death such as cancer [10], coronary heart disease (CHD) [11] and all-cause death $[12,13]$. Current studies addressing the relatonship between the NLR and the risk of fatal stroke focused mainly on stroke onset, and corresponding results are inconsistent because of no long period enough for conducting follow-up [14-16]. Such data and information were based on acute inpatients, and all of tests were conducted after stroke happened. Thus, these results should reflect a status under stress reaction, and the NLR should be more likely to direct an acute inflammation. Although the NLR could be used as a predictor for future mortality risk of CHD [11], it is unclear whether the NLR is associated with the risk of fatal stroke occurrence. Here, we aimed to systematically assess the relationship between the NLR and future risk of fatal stroke, fatal ischaemic or haemorrhagic stroke occurrence in a relatively healthy older population.

\section{Methods}




\section{Data source and participants}

All GBCS (Guangzhou Biobank Cohort Study) participants were recruited from a population of permanent residents aged 50 years or older in Guangzhou in southern China. Details of the GBCS have been reported previously [17]. The baseline (from September $30^{\text {th }}, 2003$ to February $28^{\text {th }}, 2008$ ) and follow-up information included a face-to-face computer-assisted interview by trained nurses on lifestyle, the family and personal medical history ; assessments of anthropometric data and blood pressure; and a series of laboratory tests. Each participant had made an appointment in advance to ensure good health, was able to come the designated place, and was able to sit and rest for at least half an hour before sampling and examination.

\section{Exposure indicators}

The blood cell counts were performed with a counter (KX-21, Sysmex, Japan) in Guangzhou Twelfth People's Hospital. The neutrophil (NEUT) and the lymphocyte (LYM) counts were determined sautomatically. The NLR was calculated accordingly from the NEUT and the LYM counts. Fasting glucose, cholesterol, triglycerides, liver and kidney function and CRP were measured with an analyser (Cobas c-311, Roche, Switzerland). The hospital laboratory performs internal and external quality control procedures according to the China Association of Laboratory Quality Control.

\section{Study outcomes and potential confounders}

Information on underlying causes of death up to December $31^{\text {st }}, 2017$, was obtained mostly via record linkage with the Guangzhou Centers for Disease Control and Prevention (GZCDC). Because there was no other information for stroke severity, infarct volume, site of lesion and infectious complications, fatal stroke occurrence was chosen as the primary outcome of this study. Death causes were coded according to the $10^{\text {th }}$ revision of the International Classification of Diseases (ICD) as follows: I60-169 for stroke; I60.0-162.9 and I69.0-169.2 for haemorrhagic stroke; I63.0-I63.9 and I69.3 for ischaemic stroke; and the other codes for unclassified stroke. When the death certificates were not issued by medical institutions, the causes were verified by the GZCDC as part of their quality assurance programmed by cross-checking past medical history and conducting verbal autopsy by 5 senior clinicians from Guangzhou Twelfth People's Hospital, the Universities of Hong Kong, China and Birmingham, UK. To examine the extent to which baseline factors in relation to the risks of stroke, ischaemic and haemorrhagic stroke, we included the factors in different models. Model 1 was a crude hazard ratio model without adjustment for any confounders. Model 2 contained a multivariate adjustment for factors including sex, age, education (primary and below, middle school, and college or above), occupation (manual, nonmanual, and others), smoking (never, former and current), alcohol consumption (never, former and current), International Physical Activity Questionnaire-assessed physical activity (inactive, moderate and active) [18], body mass index (BMI, defined as

weight in $\mathrm{kg} \div$ eight in $\mathrm{m}^{2}$ ), self-rated health, hypertension, diabetes, dyslipidaemia, cancer, genitourinary disease, chest disease and the platelet count. Model 3 included CRP as a competing confounder in addition to the confounders in model 2.

\section{Statistical analysis}

The NEUTs, the LYMs and the NLRs were classified by quartiles, respectively: the $1^{\text {st }}$ quartile $\left(<3.0^{\star} 10^{\wedge} 9 / \mathrm{L}\right)$, the $2^{\text {nd }}$ quartile $\left(3.0-3.6^{\star} 10^{\wedge} 9 / \mathrm{L}\right)$, the $3^{\text {rd }}$ quartile $\left(3.7-4.4^{\star} 10^{\wedge} 9 / \mathrm{L}\right)$ and the $4^{\text {th }}$ quartile $\left(>4.5^{\star} 10^{\wedge} 9 / \mathrm{L}\right)$ for the NEUTs; the $1^{\text {st }}$ quartile $\left(<1.8^{\star} 10^{\wedge} 9 / \mathrm{L}\right)$, the $2^{\text {nd }}$ quartile $\left(1.8-2.1^{\star} 10^{\wedge} 9 / \mathrm{L}\right)$, the $3^{\text {rd }}$ quartile $\left(2.2-2.5^{\star} 10^{\wedge} 9 / \mathrm{L}\right)$ and the $4^{\text {th }}$ quartile $(>$ $\left.2.5^{\star} 10^{\wedge} 9 / \mathrm{L}\right)$ for the LYM; the $1^{\text {st }}$ quartile $(\leq 1.39)$, the $2^{\text {nd }}$ quartile $(1.40-1.75)$, the $3^{\text {rd }}$ quartile $(1.76-2.23)$ and the $4^{\text {th }}$ 
quartile ( $\geq 2.24$ ) for the NLR; and the NLR was then analysed as a continuous parameter using a restricted cubic splines curve model (RCS), and as a dichotomous variable and continuous variable respectively: 3 knots at the $10^{\text {th }}$, the $50^{\text {th }}$, and the $90^{\text {th }}$ percentiles for the RCS; $\leq 1.75$ versus $>1.75$ for a dichotomous variable. Continuous variables are presented as the mean \pm standard deviation, and categorical variables were described by frequency and percentage. The chi-squared and Fisher's exact test were used for categorical variables, and analysis of variance (ANOVA) and the Kruskal-Wallis test were used for continuous variables. Based on the results of the crude hazard ratio model analysis, a sensitivity analysis was conducted in which model 2 and model 3 were repeated for the participants without CVD at baseline and further adjustment for CRP. All analyses were performed using STATA (Version 14.0; StataCorp LP, College Station, TX, USA). All $p$ values were 2 sided, and statistical significance was defined as $p<0.05 ; p$ values for trends in the models were calculated as ordinal scores from the $2^{\text {nd }}$, the $3^{\text {rd }}$ and the $4^{\text {th }}$ quartiles when taking the $1^{\text {st }}$ quartile as the reference.

\section{Results}

\section{Baseline characteristics}

In total, 30,430 participants were screened. Among the data exclusions, there were 286 because of a previous history of stroke, 315 because of an unclear stroke history, 372 because of loss to follow-up with unknown vital status, and 1646 because of incomplete information on the NEUT, the LYM and platelet counts, hypertension, diabetes, dyslipidaemia, smoking, alcohol consumption, physical activity, BMI, self-rated health, cancer, genitourinary disease or chest disease. A total of 27,811 participants who were free of stroke at baseline were included in this study. After a mean follow-up time of 11.5 (standard deviation = 2.3) years with 320,859 person-years, 503 stroke deaths (227 ischaemic, 172 haemorrhagic and 104 unclassified) were recorded (Fig. 1).

The baseline characteristics of the participants are presented in Table 1. Compared to the population in the 1st NLR quartile ( $\leq 1.39)$, the population in the highest NLR $(\geq 2.24)$ included more men, were older, had more hypertensive, current smoking and drinker, diabetes; had less physical activity, dyslipidemia, genitourinary disease and cancer, and had poorer self-rated health. 
Table 1

Baseline characteristics by the NLR quartiles of participants in the GBCS $(n=27811)$

\begin{tabular}{|c|c|c|c|c|c|}
\hline \multirow[t]{3}{*}{ Characteristic } & \multicolumn{4}{|l|}{ Quartile of NLR } & \multirow[t]{3}{*}{$P$} \\
\hline & the 1st & the 2nd & the 3rd & the 4th & \\
\hline & $(\leq 1.39)$ & $(1.40-1.752)$ & $(1.76-2.23)$ & $(\geq 2.24)$ & \\
\hline Number, $\mathrm{n}$ & 7032 & 6992 & 6762 & 7025 & \\
\hline Age (years) & $60.8 \pm 6.7$ & $61.5 \pm 6.9$ & $62.2 \pm 7.1$ & $63.5 \pm 7.4$ & $<0.001$ \\
\hline Sex, male (\%) & $1336(19 \%)$ & $1670(23.9 \%)$ & $1941(28.7 \%)$ & $2684(38.2 \%)$ & $<0.001$ \\
\hline Hypertension, n (\%) & $1726(24.5 \%)$ & $1891(27 \%)$ & 2017 (29.8\%) & $2182(31.1 \%)$ & $<0.001$ \\
\hline Diabetes, n (\% ) & $782(11.1 \%)$ & $853(12.2 \%)$ & $925(13.7 \%)$ & $1069(15.2 \%)$ & $<0.001$ \\
\hline Dyslipidemia, n (\%) & $5983(85.1 \%)$ & $5821(83.3 \%)$ & $5613(83 \%)$ & $5607(79.8 \%)$ & $<0.001$ \\
\hline Smoking, n (\%) & & & & & $<0.001$ \\
\hline never & $6090(86.6 \%)$ & $5875(84 \%)$ & $5385(79.6 \%)$ & $5175(73.7 \%)$ & \\
\hline ever & $476(6.8 \%)$ & $541(7.8 \%)$ & $623(9.2 \%)$ & $882(12.5 \%)$ & \\
\hline current & $466(6.6 \%)$ & $576(8.2 \%)$ & $754(11.2 \%)$ & $968(13.8 \%)$ & \\
\hline Alcohol drinking, n (\%) & & & & & $<0.001$ \\
\hline never & $5056(71.9 \%)$ & 4969 (71.1\%) & $4700(69.5 \%)$ & $4816(68.6 \%)$ & \\
\hline ever & $132(1.9 \%)$ & $141(2 \%)$ & $156(2.3 \%)$ & $212(3 \%)$ & \\
\hline current & $1844(26.2 \%)$ & $1882(26.9 \%)$ & $1906(28.2 \%)$ & 1997 (28.4\%) & \\
\hline Body mass index, $\mathrm{kg} / \mathbb{\nabla}$ & & & & & $<0.001$ \\
\hline$<18.5$ & $280(4 \%)$ & $275(3.9 \%)$ & $281(4.2 \%)$ & $410(5.8 \%)$ & \\
\hline $18.5-23.9$ & 3598 (51.1\%) & $3441(49.2 \%)$ & 3347 (49.5\%) & $3652(50.7 \%)$ & \\
\hline $24-27.9$ & $2480(35.3 \%)$ & 2549 (36.5\%) & $2436(36 \%)$ & $2392(34.1 \%)$ & \\
\hline$\geq 28$ & $674(9.6 \%)$ & $727(10.4 \%)$ & $698(10.3 \%)$ & $661(9.4 \%)$ & \\
\hline Physical activity, n (\%) & & & & & $<0.001$ \\
\hline inactive & $610(8.7 \%)$ & $564(8.1 \%)$ & $525(7.8 \%)$ & $556(7.9 \%)$ & \\
\hline moderate & $2712(38.5 \%)$ & $2806(40.1 \%)$ & $2797(41.3 \%)$ & $3031(43.2 \%)$ & \\
\hline active & $3710(52.8 \%)$ & $3622(51.8 \%)$ & $3440(50.9 \%)$ & $3438(48.9 \%)$ & \\
\hline Self-rated health, $\mathrm{n}(\%)$ & $5880(83.6 \%)$ & $5847(83.6 \%)$ & $5539(81.9 \%)$ & $5711(81.3 \%)$ & $<0.001$ \\
\hline (good/very good) & & & & & \\
\hline
\end{tabular}

Hypertension: systolic blood pressure, $\geq 140 \mathrm{mmHg}$, diastolic blood pressure, $\leq 90 \mathrm{mmHg}$, medication or diagnoseis; diabetes: fasting blood glucose $\geq 7$, medication or diagnosis; dyslipidemia: total cholesterol $\geq 5.2 \mathrm{mmol} / \mathrm{L}$, triglyceride $\geq 1.7 \mathrm{mmol} / \mathrm{L}$, low density lipoprotein $\geq 3.4 \mathrm{mmol} / \mathrm{L}$, high density lipoprotein $<1.0 \mathrm{mmol} / \mathrm{L}$, medication or diagnosis; hsCRP: high-sensitivity C-reactive protein; NLR: neutrophil to lymphocyte ratio; NEUT: neutrophil; Lym: lymphocyte; GD: Genitourinary disease (including nephropathy, prostatic disease, and gynecologic diseases); chest disease: including COPD, chronic bronchitis, emphysema, asthma, tuberculosis, and pneumonia. 


\begin{tabular}{|c|c|c|c|c|c|}
\hline \multirow[t]{3}{*}{ Characteristic } & \multicolumn{4}{|l|}{ Quartile of NLR } & \multirow[t]{3}{*}{$P$} \\
\hline & the 1st & the 2nd & the 3rd & the 4th & \\
\hline & $(\leq 1.39)$ & $(1.40-1.752)$ & $(1.76-2.23)$ & $(\geq 2.24)$ & \\
\hline Cancer, n (\% ) & $152(2.2 \%)$ & $141(2 \%)$ & $120(1.8 \%)$ & $126(1.8 \%)$ & 0.28 \\
\hline GD, n (\% ) & 1995 (28.4\%) & $1906(27.3 \%)$ & $1732(25.6 \%)$ & $1772(25.2 \%)$ & $<0.001$ \\
\hline Chest disease, $\mathrm{n}(\%)$ & $1092(15.5 \%)$ & $1004(14.4 \%)$ & 1009 (14.9\%) & $1108(15.8 \%)$ & 0.09 \\
\hline NEUT, *10^9/L & $2.8 \pm 0.78$ & $3.5 \pm 0.80$ & $4.0 \pm 0.93$ & $5.0 \pm 1.68$ & $<0.001$ \\
\hline Lym, *10^9/L & $2.5 \pm 0.64$ & $2.2 \pm 0.50$ & $2.0 \pm 0.47$ & $1.7 \pm 0.44$ & $<0.001$ \\
\hline Platelet, *10^9/L & $221.4 \pm 61$ & $226.5 \pm 56.7$ & $228.4 \pm 56$ & $233.1 \pm 66.1$ & $<0.001$ \\
\hline hsCRP, mg/L & $2.9 \pm 2.5$ & $3.2 \pm 2.6$ & $3.7 \pm 2.8$ & $4.2 \pm 3.2$ & $<0.001$ \\
\hline \multicolumn{6}{|c|}{$\begin{array}{l}\text { Hypertension: systolic blood pressure, } \geq 140 \mathrm{mmHg} \text {, diastolic blood pressure, } \leq 90 \mathrm{mmHg} \text {, medication or diagnoseis; } \\
\text { diabetes: fasting blood glucose } \geq 7 \text {, medication or diagnosis; dyslipidemia: total cholesterol } \geq 5.2 \mathrm{mmol} / \mathrm{L} \text {, } \\
\text { triglyceride } \geq 1.7 \mathrm{mmol} / \mathrm{L} \text {, low density lipoprotein } \geq 3.4 \mathrm{mmol} / \mathrm{L} \text {, high density lipoprotein }<1.0 \mathrm{mmol} / \mathrm{L} \text {, medication or } \\
\text { diagnosis; hsCRP: high-sensitivity C-reactive protein; NLR: neutrophil to lymphocyte ratio; NEUT: neutrophil; Lym: } \\
\text { lymphocyte; GD: Genitourinary disease (including nephropathy, prostatic disease, and gynecologic diseases); chest } \\
\text { disease: including COPD, chronic bronchitis, emphysema, asthma, tuberculosis, and pneumonia. }\end{array}$} \\
\hline
\end{tabular}

\section{TheNEUT and the LYM in relation to the risk of fatal stroke occurrence}

Prior to the NLR analysis, we observed that those in the highest NEUT quartile had a significant association with an increased risk for fatal stroke $(\mathrm{aHR}=1.45,95 \% \mathrm{Cl} 1.10-1.89, P=0.008)$ and fatal ischaemic stroke $(\mathrm{aHR}=1.65,95 \% \mathrm{Cl}$ 1.10-2.47, $P=0.02$ ), while no significant association was obtained between the LYM and the risk of fatal stroke after adjustments for a series of factors (Table 2). 
Table 2

Association between the neutrophil and the lymphocyte counts and the risk of fatal stroke in the GBCS, 2003-2017 ( $\mathrm{n}=$ 27811)

\begin{tabular}{|c|c|c|c|c|c|c|c|c|c|c|}
\hline \multirow{2}{*}{$\begin{array}{l}\text { Pvalue } \\
\text { trend }\end{array}$} & \multicolumn{4}{|c|}{ Quartiles of NEUT $\left(* 10^{\wedge} 9 / \mathrm{L}\right)$} & \multirow{2}{*}{$\begin{array}{l}P \\
\text { value } \\
\text { trend }\end{array}$} & \multicolumn{4}{|c|}{ Quartiles of LYM (*10^9/L) } & \multirow{2}{*}{$\begin{array}{l}P \\
\text { value } \\
\text { trend }\end{array}$} \\
\hline & $\begin{array}{l}\text { the } \\
\text { 1st } \\
(<3.0)\end{array}$ & $\begin{array}{l}\text { the 2nd } \\
(3.0-3.6)\end{array}$ & $\begin{array}{l}\text { the 3rd } \\
(3.7-4.4)\end{array}$ & $\begin{array}{l}\text { the } \\
\text { 4th } \\
(>4.5)\end{array}$ & & $\begin{array}{l}\text { the } 1^{s} \\
{ }^{t}(<1.8)\end{array}$ & $\begin{array}{l}\text { the } \\
\text { 2nd } \\
(1.8- \\
2.1)\end{array}$ & $\begin{array}{l}\text { the } \\
3 r d \\
(2.2- \\
2.5)\end{array}$ & $\begin{array}{l}\text { the } \\
\text { 4th } \\
(>2.5)\end{array}$ & \\
\hline \multicolumn{11}{|l|}{ Stroke } \\
\hline $\begin{array}{l}\text { Person } \\
\text { years }\end{array}$ & 79448 & 83038 & 77517 & 80857 & & 86199 & 93678 & 73358 & 67624 & \\
\hline $\begin{array}{l}\text { per } \\
10^{\wedge} 5 \\
\text { person- } \\
\text { years }\end{array}$ & 109.5 & 116.8 & 152.2 & 248.6 & & 178.7 & 156.9 & 140.4 & 146.4 & \\
\hline $\begin{array}{l}\text { No. of } \\
\text { deaths }\end{array}$ & 87 & 97 & 118 & 201 & & 154 & 147 & 103 & 99 & \\
\hline $\begin{array}{l}\text { Model } \\
1 \text { (HR; } \\
95 \% \\
\mathrm{Cl})\end{array}$ & Ref. & $\begin{array}{l}1.06 \\
(0.79- \\
1.41)\end{array}$ & $\begin{array}{l}1.38 \\
(1.05- \\
1.82)^{a}\end{array}$ & $\begin{array}{l}2.25 \\
(1.75- \\
2.90)^{c}\end{array}$ & $<0.001$ & Ref. & $\begin{array}{l}0.88 \\
(0.70- \\
1.10)\end{array}$ & $\begin{array}{l}0.78 \\
(0.61- \\
1.00)\end{array}$ & $\begin{array}{l}0.82 \\
(0.64- \\
1.06)\end{array}$ & 0.07 \\
\hline$P$ value & & 0.70 & 0.02 & $<0.001$ & & & 0.26 & 0.05 & 0.13 & \\
\hline $\begin{array}{l}\text { Model } \\
2 \text { (HR; } \\
95 \% \\
\mathrm{Cl})\end{array}$ & Ref. & $\begin{array}{l}0.87 \\
(0.65- \\
1.17)\end{array}$ & $\begin{array}{l}1.06 \\
(0.80- \\
1.41)\end{array}$ & $\begin{array}{l}1.45 \\
(1.10- \\
1.89)^{\mathrm{b}}\end{array}$ & 0.001 & Ref. & $\begin{array}{l}1.01 \\
(0.80- \\
1.27)\end{array}$ & $\begin{array}{l}0.92 \\
(0.71- \\
1.19)\end{array}$ & $\begin{array}{l}0.95 \\
(0.73- \\
1.24)\end{array}$ & 0.56 \\
\hline$P$ value & & 0.36 & 0.68 & 0.008 & & . & 0.92 & 0.51 & 0.71 & \\
\hline \multicolumn{11}{|c|}{ Ischaemic stroke } \\
\hline $\begin{array}{l}\text { Person } \\
\text { years }\end{array}$ & 79045 & 82625 & 76875 & 80068 & & 85536 & 93017 & 72896 & 67163 & \\
\hline $\begin{array}{l}\text { per } \\
10^{\wedge} 5 \\
\text { person- } \\
\text { years }\end{array}$ & 46.8 & 54.5 & 61.1 & 122.4 & & 86.5 & 77.4 & 52.1 & 64.0 & \\
\hline $\begin{array}{l}\text { No. of } \\
\text { deaths }\end{array}$ & 37 & 45 & 47 & 98 & & 74 & 72 & 38 & 43 & \\
\hline $\begin{array}{l}\text { Model } \\
1 \text { (HR; } \\
95 \% \\
\mathrm{Cl})\end{array}$ & Ref. & $\begin{array}{l}1.16 \\
(0.75- \\
1.78)\end{array}$ & $\begin{array}{l}1.29 \\
(0.84- \\
1.99)\end{array}$ & $\begin{array}{l}2.59 \\
(1.78- \\
3.79)^{c}\end{array}$ & $<0.001$ & Ref. & $\begin{array}{l}0.90 \\
(0.65- \\
1.24)\end{array}$ & $\begin{array}{l}0.60 \\
(0.41- \\
0.89)^{a}\end{array}$ & $\begin{array}{l}0.75 \\
(0.51- \\
1.09)\end{array}$ & 0.03 \\
\hline$P$ value & & 0.52 & 0.24 & $<0.001$ & & & 0.51 & 0.01 & 0.13 & \\
\hline
\end{tabular}

Ref: reference; ${ }^{\mathrm{C}} P<0.001,{ }^{\mathrm{b}} P<0.01,{ }^{\mathrm{a}} P<0.05$; model 1 : a crude hazard ratio model without adjustment for confounders; model 2: a multivariate model adjusted for sex, age, education, occupation, diabetes, hypertension, dyslipidaemia, smoking, alcohol consumption, physical activity, body mass index, self-rated health, cancer, genitourinary disease (including nephropathy, prostatic disease, and gynaecologic diseases), chest disease (including chronic obstructive pulmonary disease, chronic bronchitis, emphysema, asthma, tuberculosis, and pneumonia) and platelet count. 


\begin{tabular}{|c|c|c|c|c|c|c|c|c|c|c|}
\hline \multirow{3}{*}{$\begin{array}{l}\text { Pvalue } \\
\text { trend }\end{array}$} & \multicolumn{4}{|c|}{ Quartiles of NEUT (*10^9/L) } & \multirow{3}{*}{$\begin{array}{l}P \\
\text { value } \\
\text { trend }\end{array}$} & \multicolumn{4}{|c|}{ Quartiles of LYM (*10^9/L) } & \multirow{3}{*}{$\begin{array}{l}P \\
\text { value } \\
\text { trend }\end{array}$} \\
\hline & $\begin{array}{l}\text { the } \\
1 \text { st }\end{array}$ & $\begin{array}{l}\text { the 2nd } \\
(3.0-3.6)\end{array}$ & $\begin{array}{l}\text { the 3rd } \\
(3.7-4.4)\end{array}$ & $\begin{array}{l}\text { the } \\
\text { 4th }\end{array}$ & & \multirow{2}{*}{$\begin{array}{l}\text { the } 1^{s} \\
{ }^{t}(<1.8)\end{array}$} & \multirow{2}{*}{$\begin{array}{l}\text { the } \\
2 \text { nd } \\
(1.8- \\
2.1)\end{array}$} & \multirow{2}{*}{$\begin{array}{l}\text { the } \\
3 \text { rd } \\
(2.2- \\
2.5)\end{array}$} & \multirow{2}{*}{$\begin{array}{l}\text { the } \\
\text { 4th } \\
(>2.5)\end{array}$} & \\
\hline & $(<3.0)$ & & & $(>4.5)$ & & & & & & \\
\hline Model & Ref. & 0.95 & & & 0.004 & Ref. & 1.05 & 0.71 & 0.89 & 0.24 \\
\hline $\begin{array}{l}95 \% \\
\mathrm{Cl})\end{array}$ & & $\begin{array}{l}(0.61- \\
1.48)\end{array}$ & $1.55)$ & $2.47)^{a}$ & & & $\begin{array}{l}(0.75- \\
1.45)\end{array}$ & $\begin{array}{l}(0.48- \\
1.07)\end{array}$ & $\begin{array}{l}(0.60- \\
1.32)\end{array}$ & \\
\hline$P$ value & & 0.83 & 0.99 & 0.02 & & & 0.80 & 0.10 & 0.56 & \\
\hline \multicolumn{11}{|c|}{ Haemorrhagic stroke } \\
\hline $\begin{array}{l}\text { Person } \\
\text { years }\end{array}$ & 79017 & 82461 & 76779 & 79663 & & 85280 & 92716 & 72823 & 67083 & \\
\hline $\begin{array}{l}\text { per } \\
10^{\wedge} 5 \\
\text { person- } \\
\text { years }\end{array}$ & 50.6 & 37.6 & 48.2 & 82.8 & & 64.5 & 43.1 & 57.7 & 52.2 & \\
\hline $\begin{array}{l}\text { No. of } \\
\text { deaths }\end{array}$ & 40 & 31 & 37 & 66 & & 55 & 40 & 42 & 35 & \\
\hline \multirow{2}{*}{$\begin{array}{l}\text { Model } \\
1(\mathrm{HR} ; \\
95 \% \\
\mathrm{Cl})\end{array}$} & \multirow[t]{2}{*}{ Ref. } & 0.74 & \multirow{2}{*}{$\begin{array}{l}0.94 \\
(0.60- \\
1.47)\end{array}$} & \multirow{2}{*}{$\begin{array}{l}1.61 \\
(1.09- \\
2.39)^{a}\end{array}$} & \multirow[t]{2}{*}{0.004} & \multirow[t]{2}{*}{ Ref. } & 0.67 & 0.89 & 0.81 & \multirow[t]{2}{*}{0.55} \\
\hline & & $\begin{array}{l}(0.46- \\
1.18)\end{array}$ & & & & & $\begin{array}{l}(0.45- \\
1.01)\end{array}$ & $\begin{array}{l}(0.60- \\
1.33)\end{array}$ & $\begin{array}{l}(0.53- \\
1.24)\end{array}$ & \\
\hline \multicolumn{2}{|l|}{$P$ value } & 0.20 & 0.79 & 0.02 & & & 0.05 & 0.57 & 0.34 & \\
\hline \multirow{2}{*}{$\begin{array}{l}\text { Model } \\
2 \text { (HR; } \\
95 \% \\
\mathrm{Cl})\end{array}$} & \multirow[t]{2}{*}{ Ref. } & 0.63 & \multirow{2}{*}{$\begin{array}{l}0.77 \\
(0.49- \\
1.22)\end{array}$} & \multirow{2}{*}{$\begin{array}{l}1.14 \\
(0.74- \\
1.75)\end{array}$} & \multirow[t]{2}{*}{0.26} & \multirow[t]{2}{*}{ Ref. } & 0.77 & 1.03 & 0.94 & \multirow[t]{2}{*}{0.94} \\
\hline & & $\begin{array}{l}(0.39- \\
1.02)\end{array}$ & & & & & $\begin{array}{l}(0.51- \\
1.16)\end{array}$ & $\begin{array}{l}(0.68- \\
1.57)\end{array}$ & $\begin{array}{l}(0.60- \\
1.46)\end{array}$ & \\
\hline$P$ value & & 0.06 & 0.27 & 0.56 & & & 0.21 & 0.88 & 0.77 & \\
\hline \multicolumn{11}{|c|}{$\begin{array}{l}\text { Ref: reference; }{ }^{\mathrm{C}} P<0.001,{ }^{\mathrm{b}} P<0.01,{ }^{\mathrm{a}} P<0.05 \text {; model } 1 \text { : a crude hazard ratio model without adjustment for } \\
\text { confounders; model } 2 \text { : a multivariate model adjusted for sex, age, education, occupation, diabetes, hypertension, } \\
\text { dyslipidaemia, smoking, alcohol consumption, physical activity, body mass index, self-rated health, cancer, } \\
\text { genitourinary disease (including nephropathy, prostatic disease, and gynaecologic diseases), chest disease (including } \\
\text { chronic obstructive pulmonary disease, chronic bronchitis, emphysema, asthma, tuberculosis, and pneumonia) and } \\
\text { platelet count. }\end{array}$} \\
\hline
\end{tabular}

\section{The NLR in relation to the risk of fatal stroke occurrence}

In a restricted cubic splines model, there is an increased trend of the relationship between the NLR and the risk of fatal stroke occurrence, and the cutoff value of NLR level was of 1.19 after adjustments were made in a multivariable adjustment analysis (Fig. 2). Both Table 3 and Fig. 3 shows the NLRs in relation to the risk of fatal stroke occurrence. After adjustment for a series of factors, the NLRs were associated an increased risk of fatal stroke and fatal ischaemic stroke (all $P$ for trend $<0.001)$; the participants in the 4th NLR quartile $(\geq 2.24)$ had an increased risk of fatal stroke (adjusted HR $(\mathrm{aHR})=1.76,95 \% \mathrm{Cl} 1.33-2.32, P<0.001)$ and fatal ischaemic stroke $(\mathrm{aHR}=2.15,95 \% \mathrm{Cl} 1.41-3.28, P<$ 0.001), respectively. Similar results were observed for fatal stroke (men: aHR $=1.96,95 \% \mathrm{Cl} 1.24-3.08, P=0.004$; women: $\mathrm{aHR}=1.57,95 \% \mathrm{Cl} 1.10-2.25, P=0.01)$ and fatal ischaemic stroke (men: aHR $=2.23,95 \% \mathrm{Cl} 1.17-4.27, P=0.02$; women: $\mathrm{aHR}=2.01,95 \% \mathrm{Cl} 1.15-3.53, P=0.02)$. Table 4 shows higher NLR in relation to an increased risk of fatal stroke $(\mathrm{aHR}=$ 
$1.89,95 \% \mathrm{Cl} 1.26-2.82, P=0.002)$, fatal ischaemic stroke $(\mathrm{aHR}=3.34,95 \% \mathrm{Cl} 1.73-6.45, P<0.001)$; and an increasing trend was obtained for fatal stroke $(P<0.001)$ and fatal ischaemic stroke $(P<0.001)$ after further CRP adjustment. 
Table 3

Association between the NLRs and the risk of fatal stroke occurrence in the GBCS $(n=27811)$

\section{Quartiles of NLR}

$\begin{array}{llll}\text { the } 1 \mathrm{st} & \text { the } 2 \mathrm{nd} & \text { the } 3 \mathrm{rd} & \text { the } 4 \text { th } \\ (\leq & (1.40-1.752) & (1.76-2.23) & (\geq 2.24) \\ 1.39) & & & \end{array}$

Stroke

overall

\begin{tabular}{|c|c|c|c|c|}
\hline $\begin{array}{l}\text { Model } 1 \text { (HR, 95\% } \\
\mathrm{Cl})\end{array}$ & 1.00 & $\begin{array}{l}1.33(0.98-1.80), P= \\
0.07\end{array}$ & $\begin{array}{l}2.08(1.57-2.77), P< \\
0.001\end{array}$ & $\begin{array}{l}2.78(2.12-3.65), P< \\
0.001\end{array}$ \\
\hline $\begin{array}{l}\text { Model } 2 \text { (HR, 95\% } \\
\mathrm{Cl})\end{array}$ & 1.00 & $\begin{array}{l}1.19(0.87-1.62), P= \\
0.27\end{array}$ & $\begin{array}{l}1.61(1.21-2.15), P= \\
0.001\end{array}$ & $\begin{array}{l}1.76(1.33-2.32), P< \\
0.001\end{array}$ \\
\hline
\end{tabular}

$P$ for trend $\quad<0.001$

men

\begin{tabular}{|c|c|c|c|c|}
\hline $\begin{array}{l}\text { Model } 1 \text { (HR, 95\% } \\
\mathrm{Cl})\end{array}$ & 1.00 & $\begin{array}{l}1.04(0.61-1.78), P= \\
0.89\end{array}$ & $\begin{array}{l}1.80(1.11-2.91), P= \\
0.02\end{array}$ & $\begin{array}{l}2.37(1.51-3.71), P< \\
0.001\end{array}$ \\
\hline $\begin{array}{l}\text { Model } 2 \text { (HR, 95\% } \\
\mathrm{Cl})\end{array}$ & 1.00 & $\begin{array}{l}1.07(0.62-1.83), P= \\
0.82\end{array}$ & $\begin{array}{l}1.71(1.05-2.76), P= \\
0.03\end{array}$ & $\begin{array}{l}1.96(1.24-3.08), P= \\
0.004\end{array}$ \\
\hline
\end{tabular}

$P$ for trend $\quad<0.001$

women

\begin{tabular}{|c|c|c|c|c|}
\hline $\begin{array}{l}\text { Model } 1 \text { (HR, 95\% } \\
\text { Cl) }\end{array}$ & 1.00 & $\begin{array}{l}1.41(0.97-2.05), P= \\
0.07\end{array}$ & $\begin{array}{l}1.99(1.40-2.85), P< \\
0.001\end{array}$ & $\begin{array}{l}2.36(1.66-3.36), P< \\
0.001\end{array}$ \\
\hline $\begin{array}{l}\text { Model } 2 \text { (HR, 95\% } \\
\mathrm{Cl})\end{array}$ & 1.00 & $\begin{array}{l}1.26(0.87-1.84), P= \\
0.23\end{array}$ & $\begin{array}{l}1.58(1.10-2.26), P= \\
0.01\end{array}$ & $1.57(1.10-2.25), P=0.01$ \\
\hline
\end{tabular}

$P$ for trend $\quad 0.007$

Ischemic stroke

overall

\begin{tabular}{|c|c|c|c|c|}
\hline $\begin{array}{l}\text { Model } 1 \text { (HR, 95\% } \\
\mathrm{Cl})\end{array}$ & 1.00 & $\begin{array}{l}1.50(0.94-2.40), P= \\
0.09\end{array}$ & $\begin{array}{l}1.86(1.18-2.93), P= \\
0.008\end{array}$ & $\begin{array}{l}3.59(2.38-5.42), P< \\
0.001\end{array}$ \\
\hline $\begin{array}{l}\text { Model } 2 \text { (HR, 95\% } \\
\mathrm{Cl})\end{array}$ & 1.00 & $\begin{array}{l}1.34(0.84-2.14), P= \\
0.22\end{array}$ & $\begin{array}{l}1.40(0.88-2.20), P= \\
0.15\end{array}$ & $\begin{array}{l}2.15(1.41-3.28), P< \\
0.001\end{array}$ \\
\hline
\end{tabular}

$P$ for trend $\quad<0.001$

men

\begin{tabular}{|c|c|c|c|c|}
\hline $\begin{array}{l}\text { Model } 1 \text { (HR, 95\% } \\
\text { Cl) }\end{array}$ & 1.00 & $\begin{array}{l}1.05(0.48-2.29), P= \\
0.90\end{array}$ & $\begin{array}{l}1.73(0.86-3.47), P= \\
0.13\end{array}$ & $\begin{array}{l}2.73(1.43-5.20), P= \\
0.002\end{array}$ \\
\hline $\begin{array}{l}\text { Model } 2(\mathrm{HR}, 95 \% \\
\mathrm{Cl})\end{array}$ & 1.00 & $\begin{array}{l}1.06(0.49-2.31), P= \\
0.89\end{array}$ & $\begin{array}{l}1.59(0.79-3.21), P= \\
0.19\end{array}$ & $2.23(1.17-4.27)$ \\
\hline
\end{tabular}

NLR: neutrophil to lymphocyte ratio; model 1: a crude hazard ratio model without adjustments; model 2: a multivariate model adjusted for age, sex, diabetes, hypertension, dyslipidemia, smoking, alcohol consumption, physical activity, body mass index, self-rated health, cancer, genitourinary disease (nephropathy, prostatic disease, and gynecologic diseases) and chest disease (COPD, chronic bronchitis, emphysema, asthma, tuberculosis, and pneumonia) and platelet count. 


\section{Quartiles of NLR}

Pfor trend $\quad 0.002$

women

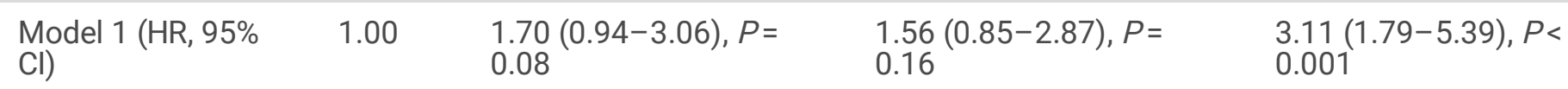

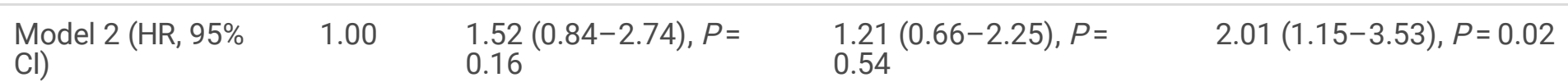

Pfor trend $\quad 0.029$

Hemorrhagic stroke

overall

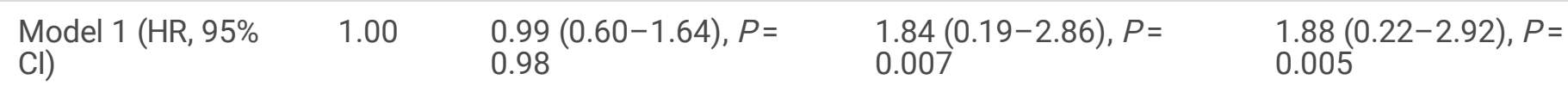

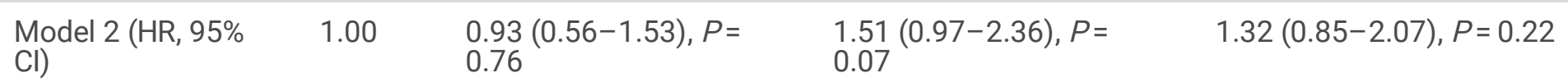

$P$ for trend $\quad 0.071$

men

\begin{tabular}{|c|c|c|c|c|}
\hline $\begin{array}{l}\text { Model } 1(\mathrm{HR}, 95 \% \\
\mathrm{Cl})\end{array}$ & 1.00 & $\begin{array}{l}1.03(0.36-2.97), P= \\
0.95\end{array}$ & $\begin{array}{l}2.05(0.82-5.18), P= \\
0.13\end{array}$ & $2.65(1.11-6.36), P=0.03$ \\
\hline $\begin{array}{l}\text { Model } 2 \text { (HR, 95\% } \\
\mathrm{Cl})\end{array}$ & 1.00 & $\begin{array}{l}1.12(0.39-3.24), P= \\
0.84\end{array}$ & $\begin{array}{l}1.99(0.79-5.04), P= \\
0.15\end{array}$ & $2.27(0.94-5.46)$ \\
\hline
\end{tabular}

$P$ for trend $\quad 0.022$

women

\begin{tabular}{|c|c|c|c|c|}
\hline $\begin{array}{l}\text { Model } 1 \text { (HR, 95\% } \\
\mathrm{Cl})\end{array}$ & 1.00 & $\begin{array}{l}0.97(0.55-1.72), P= \\
0.93\end{array}$ & $\begin{array}{l}1.73(1.04-2.88), P= \\
0.03\end{array}$ & $1.36(0.79-2.36), P=0.27$ \\
\hline $\begin{array}{l}\text { Model } 2 \text { (HR, 95\% } \\
\mathrm{Cl})\end{array}$ & 1.00 & $\begin{array}{l}0.91(0.51-1.60), P= \\
0.73\end{array}$ & $\begin{array}{l}1.43(0.86-2.38), P= \\
0.17\end{array}$ & $0.97(0.55-1.70)$ \\
\hline
\end{tabular}

$P$ for trend $\quad 0.66$

NLR: neutrophil to lymphocyte ratio; model 1: a crude hazard ratio model without adjustments; model 2: a multivariate model adjusted for age, sex, diabetes, hypertension, dyslipidemia, smoking, alcohol consumption, physical activity, body mass index, self-rated health, cancer, genitourinary disease (nephropathy, prostatic disease, and gynecologic diseases) and chest disease (COPD, chronic bronchitis, emphysema, asthma, tuberculosis, and pneumonia) and platelet count. 
Table 4

Association between the NLRs and the risk of fatal stroke occurrence among participants without CVD at baseline and further CRP adjustment $(n=10606)$

\begin{tabular}{|c|c|c|c|c|}
\hline & \multicolumn{4}{|c|}{ Quartiles of NLR } \\
\hline & the 1st & the 2nd & the 3rd & the 4th \\
\hline & $\begin{array}{l}(\leq \\
1.39)\end{array}$ & $(1.40-1.752)$ & $(1.76-2.23)$ & $(\geq 2.24)$ \\
\hline \multicolumn{5}{|l|}{ Stroke } \\
\hline $\begin{array}{l}\text { Model } 1 \text { (HR, 95\% } \\
\mathrm{Cl})\end{array}$ & 1.00 & $\begin{array}{l}1.31(0.84-2.05), P= \\
0.23\end{array}$ & $\begin{array}{l}2.07(1.37-3.11), P= \\
0.001\end{array}$ & $\begin{array}{l}2.60(1.75-3.85), P< \\
0.001\end{array}$ \\
\hline $\begin{array}{l}\text { Model } 2 \text { (HR, 95\% } \\
\text { Cl) }\end{array}$ & 1.00 & $\begin{array}{l}1.23(0.79-1.92), P= \\
0.37\end{array}$ & $1.71(1.13-2.58), P=0.01$ & $\begin{array}{l}1.89(1.26-2.82), P= \\
0.002\end{array}$ \\
\hline$P$ for trend & \multicolumn{4}{|l|}{$<0.001$} \\
\hline \multicolumn{5}{|l|}{ Ischemic stroke } \\
\hline $\begin{array}{l}\text { Model } 1(\mathrm{HR}, 95 \% \\
\mathrm{Cl})\end{array}$ & 1.00 & $\begin{array}{l}1.84(0.89-3.79), P= \\
0.10\end{array}$ & $2.37(1.18-4.74), P=0.02$ & $\begin{array}{l}4.57(2.40-8.70), P< \\
0.001\end{array}$ \\
\hline $\begin{array}{l}\text { Model } 2 \text { (HR, 95\% } \\
\mathrm{Cl})\end{array}$ & 1.00 & $\begin{array}{l}1.73(0.84-3.58), P= \\
0.14\end{array}$ & $1.98(0.98-3.97), P=0.06$ & $\begin{array}{l}3.34(1.73-6.45), P< \\
0.001\end{array}$ \\
\hline$P$ for trend & \multicolumn{4}{|l|}{$<0.001$} \\
\hline \multicolumn{5}{|l|}{ Hemorrhagic stroke } \\
\hline $\begin{array}{l}\text { Model } 1 \text { (HR, 95\% } \\
\text { Cl) }\end{array}$ & 1.00 & $\begin{array}{l}0.71(0.34-1.46), P= \\
0.35\end{array}$ & $1.54(0.85-2.81), P=0.16$ & $1.18(0.63-2.21), P=0.60$ \\
\hline $\begin{array}{l}\text { Model } 2 \text { (HR, 95\% } \\
\text { Cl) }\end{array}$ & 1.00 & $\begin{array}{l}0.67(0.32-1.38), P= \\
0.28\end{array}$ & $1.30(0.71-2.39), P=0.39$ & $0.89(0.46-1.70), P=0.71$ \\
\hline$P$ for trend & \multicolumn{4}{|l|}{0.79} \\
\hline \multicolumn{5}{|c|}{$\begin{array}{l}\text { NLR: neutrophil to lymphocyte ratio; CRP: high-sensitivity C-reactive protein; CVD: including coronary heart disease, } \\
\text { angina, myocardial infarction and peripheral vascular disease; model 1: a crude hazard ratio model without } \\
\text { adjustments; model 2: a multivariate model adjusted for age, sex, diabetes, hypertension, dyslipidemia, smoking, } \\
\text { alcohol consumption, physical activity, body mass index, self-rated health, cancer, and genitourinary disease } \\
\text { (nephropathy, prostatic disease, and gynecologic diseases), chest disease (COPD, chronic bronchitis, emphysema, } \\
\text { asthma, and tuberculosis, pneumonia), platelet count and CRP. }\end{array}$} \\
\hline
\end{tabular}

Table 5 shows the NLRs in relation to the risk of fatal stroke occurrence with another model. Compared with those in NLR $\leq 1.75$, the participants in NLRs $>1.75$ had an increased risk of fatal stroke (aHR $=1.57,95 \% \mathrm{Cl} 1.20-2.06, P=0.001)$ and fatal ischaemic stroke (aHR = 1.91, 95\% Cl 1.27-2.88, $P=0.002)$; Similar results for fatal stroke $(\mathrm{aHR}=1.1195 \% \mathrm{Cl} 1.06-$ $1.17, P<0.001)$ and fatal ischaemic stroke $(\mathrm{aHR}=1.1595 \% \mathrm{Cl} 1.09-1.21, P<0.001)$ were observed in a model conducting NLR as a continuous variable. 
Table 5

Association between the NLR categories or continuous and the risk of fatal stroke among the participants without CVD at baseline and further CRP adjustment $(n=10606)$

\section{NLR categories \\ NLR continuous}

\section{$\leq 1.75>1.75$}

\section{Stroke}

\begin{tabular}{llll} 
Model $1(\mathrm{HR}, 95 \% \mathrm{Cl})$ & 1.00 & $2.01(1.54-2.62), P<0.001$ & $1.16(1.11-1.20), P<0.001$ \\
\hline Model $2(\mathrm{HR}, 95 \% \mathrm{Cl})$ & 1.00 & $1.57(1.20-2.06), P=0.001$ & $1.11(1.06-1.17), P<0.001$
\end{tabular}

Ischemic stroke

\begin{tabular}{llll} 
Model $1(\mathrm{HR}, 95 \% \mathrm{Cl})$ & 1.00 & $2.42(1.62-3.62), P<0.001$ & $1.18(1.13-1.23), P<0.001$ \\
\hline Model $2(\mathrm{HR}, 95 \% \mathrm{Cl})$ & 1.00 & $1.91(1.27-2.88), P=0.002$ & $1.15(1.09-1.21), P<0.001$
\end{tabular}

Hemorrhagic stroke

$\begin{array}{llll}\text { Model } 1(\mathrm{HR}, 95 \% \mathrm{Cl}) & 1.00 & 1.60(1.02-2.51), P=0.04 & 1.09(0.95-1.27), P=0.23 \\ \text { Model } 2(\mathrm{HR}, 95 \% \mathrm{Cl}) & 1.00 & 1.33(0.84-2.10), P=0.23 & 1.02(0.81-1.30), P=0.85\end{array}$

NLR: neutrophil to lymphocyte ratio; CRP: high-sensitivity C-reactive protein; CVD: including coronary heart disease, angina, myocardial infarction and peripheral vascular disease; model 1: a crude hazard ratio model without adjustments; model 2: a multivariate model adjusted for age, sex, diabetes, hypertension, dyslipidemia, smoking, alcohol consumption, physical activity, body mass index, self-rated health, cancer, and genitourinary disease (nephropathy, prostatic disease, and gynecologic diseases), chest disease (COPD, chronic bronchitis, emphysema, asthma, tuberculosis, and pneumonia), platelet and CRP.

\section{Discussion}

\section{Main findings}

In this study, we found that higher NLR was associated with an increased risk of fatal stroke and fatal ischaemic stroke in a relatively healthy elderly population. The associations were independent of age, sex, hypertension, diabetes, dyslipidemia, smoking, alcohol consumption, physical activity, body mass index, self-rated health, cancer, genitourinary disease, chest disease, platelet count and CRP. This is the first report dressing the relationship between the NLR and the risk of fatal stroke occurrence in a relatively healthy older population.

\section{Current knowledge}

Among ischaemic and haemorrhagic stroke, the former accounts for more than $80 \%[19]$. In fact, chronic inflammation has come out prior to stroke onset and will follow after stroke happened [20]. Inflammatory response has also been known as a mechanism in which both the susceptibility and outcome was altered in strok [21]. The NLR has been linked to a cardiovascular or stroke disease[14-16, 22].

Atherosclerosis is accompanied by a chronic vascular wall inflammation or endothelial dysfunction, and its plaque was known as the most common cause for ischaemic stroke [23]. Neutrophils promote a rupture or erosion of atherosclerotic plaques, lead to thrombosis and trigger acute stroke events [24, 25]. The NETs (neutrophil extracellular traps) and the main components were significantly increased in acute ischaemic stroke [26] and thrombus, especially in cardiogenic cerebral embolism [27].The B2 cells (a type of lymphocyte) contributed to atherosclerosis progress, but the B1a cells (a 
type of lymphocyte) played a role of anti-atherosclerotic [28]. Higher NLR was an independent predictor for intracranial atherosclerosis [29], and was associated with an increased risk of ischaemic stroke among Korean aged 30-75 years [30], and the subjects with arterial fibrillation but without a history of stroke and transient ischaemic attack [31]. We observed not only higher NLR but also higher neutrophil was associated with an increased risk of fatal stroke and fatal ischaemic stroke, although no any significant relationship was observed between the lymphocyte and the risk of fatal stroke in this study. These results suggest a chronic inflammation has being happened prior to stroke occurrence.

\section{Implications for policy and research}

Higher NLR was related to an increased risk of fatal stroke and fatal ischaemic stroke regardless of whether a restricted cubic splines curve model or a quartile, a dichotomous variable and a continuous variable model in our study. This is the first time addressing the relationship between the NLR and the risk of fatal stroke occurrence in a relatively healthy community population. Such findings should direct a linkage of fatal stroke occurrence and a pre-existing chronic lowgrade systemic inflammation. Because the GBCS has collected a series of data from relatively healthy elders in south China, and each had been made an appointment in advance to ensure good health and was able to come the designated place by himself/herself [17]. Therefore, the fatal stroke occurrence may forewarn a burden of pre-existing chronic lowgrade systemic inflammation, especially in a large cities' elderly population.

\section{Strengths and limitations}

In our study, a mean follow-up of 11.5 years makes this a large, prospective design for a study of the general population in South China, and individuals completed a physical examination and questionnaire involving a total of 800 questions. Thus, the acquired information allows for systemic adjustments for additional potential confounding factors. However, there are limitations in this study. First, we obtained only the death information via record linkage with the GZCDC. The results of our study with death as the only outcome are obviously weakened because of the lack of analysis on other clinical outcomes of stroke events. Second, the subjects could not represent Chinese individuals due to the limitations involving the general population in South China in this study. Third, the small number of deaths limited the strength of this study to address fatal stroke, especially fatal ischaemic stroke and fatal haemorrhagic stroke. Thus, other cohorts and ethnic population should be introduced for further verified in the future.

\section{Conclusions}

Higher NLR was associated with an increase risk of fatal stroke and fatal ischaemic stroke occurrence, the clinicians should pay more attention to asymptomatic inflammatory characteristics in relatively healthy older population.

\section{Abbreviations}

NLR: neutrophil-to-lymphocyte ratio; CHD: coronary heart disease; HR: hazard ratio ; Cl: confidence interval; NEUT: neutrophil; LYM: Iymphocyte; CRP: C-reactive protein; GZCDC: Guangzhou Centers for Disease Control and Prevention; ICD: International Classification of Diseases; BMI: body mass index.

\section{Declarations}

\section{Acknowledgements}


The Guangzhou Biobank Cohort Study investigators included Guangzhou Twelfth People's Hospital: Weisen Zhang, Min Cao, Tong Zhu, Bin Liu, and Caoqiang Jiang (Co-PI); The University of Hong Kong: C.M. Schooling, S.M. McGhee, G.M. Leung, R. Fielding, and Taihing Lam (Co-PI); The University of Birmingham: P. Adab, G. Neil Thomas, and Karkeung Cheng (Co-PI).

\section{Funding}

This work was supported by the Guangzhou Science and Technology Bureau, Guangzhou, China (201704030132, 202102080467) and Guangdong Provincial Medical Science Research Foundation, Guangdong, China (A2021124). The funders had no role in the study design, data collection and analysis, the decision to publish, or the preparation of the manuscript. The work was not funded by any industry sponsors.

\section{Availability of data and materials}

The datasets used and/or analyzed in this study are available from the corresponding author on reasonable request.

\section{Ethics approval and consent to participate}

The study was approved by the Guangzhou Medical Ethics Committee of the Chinese Medical Association. All participants signed informed consent forms before participation. All methods in this study were performed in accordance with the Declaration of Helsinki.

\section{Consent for publication}

Not applicable.

\section{Competing interests}

The authors declare that they have no competing interests.

\section{Contributors}

ZBH and ZXL contributed equally to this paper for data collection and analysis. FZ contributed to the study design and wrote the manuscript. All authors reviewed the manuscript.

\section{Author details}

${ }^{1}$ Department of Internal Medicine and Central laboratory, Guangzhou Twelfth People's Hospital, No.1 Tianqiang St., Huangpu Rd., Guangzhou 510620, China. ${ }^{2}$ Department of Internal Medicine, Sanya Central Hospital, No.1154 Jiefang Rd., Sanya 572019, China. Zhi-Bing Hu: 18022868581@163.com; Ze-Xiong Lu: 1158103839@qq.com; Feng Zhu: chifengzhu@hotmail.com

\section{References}


1. Collaborators GBDM: Global, regional, and national under-5 mortality, adult mortality, age-specific mortality, and life expectancy, 1970-2016: a systematic analysis for the Global Burden of Disease Study 2016. Lancet 2017, 390(10100):1084-1150.

2. Zhou M, Wang H, Zhu J, Chen W, Wang L, Liu S, Li Y, Wang L, Liu Y, Yin P et al: Cause-specific mortality for 240 causes in China during 1990-2013: a systematic subnational analysis for the Global Burden of Disease Study 2013. Lancet 2016, 387(10015):251-272.

3. Pietri P, Vlachopoulos C, Tousoulis D: Inflammation and Arterial Hypertension: From Pathophysiological Links to Risk Prediction. Current medicinal chemistry 2015, 22(23):2754-2761.

4. Khodabandehloo H, Seyyedebrahimi S, Esfahani EN, Razi F, Meshkani R: Resveratrol supplementation decreases blood glucose without changing the circulating CD14(+)CD16(+) monocytes and inflammatory cytokines in patients with type 2 diabetes: a randomized, double-blind, placebo-controlled study. Nutrition research 2018, 54:40-51.

5. Vilahur G, Ben-Aicha S, Badimon L: New insights into the role of adipose tissue in thrombosis. Cardiovascular research 2017, 113(9):1046-1054.

6. Madani A, Alack K, Richter MJ, Kruger K: Immune-regulating effects of exercise on cigarette smoke-induced inflammation. Journal of inflammation research 2018, 11:155-167.

7. Chan JF, Yuan S, Kok KH, To KK, Chu H, Yang J, Xing F, Liu J, Yip CC, Poon RW et al: A familial cluster of pneumonia associated with the $\mathbf{2 0 1 9}$ novel coronavirus indicating person-to-person transmission: a study of a family cluster. Lancet 2020, 395(10223):514-523.

8. Wong TS, Liao KF, Lin CM, Lin CL, Chen WC, Lai SW: Chronic Pancreatitis Correlates With Increased Risk of Cerebrovascular Disease: A Retrospective Population-Based Cohort Study in Taiwan. Medicine 2016, 95(15):e3266.

9. Tseng $\mathrm{CH}$, Huang WS, Muo CH, Chang YJ, Sung FC: Increased risk of intracerebral hemorrhage among patients with chronic osteomyelitis. J Neurosurg 2015, 123(6):1528-1533.

10. Hu D, Zhang H, Lin X, Chen G, Li C, Liang B, Chen Y, Cui Z, Peng F, Zheng X et al: Elevated preoperative neutrophil-tolymphocyte ratio can predict poor survival in early stage gastric cancer patients receiving radical gastrectomy: The Fujian prospective investigation of cancer (FIESTA) study. Journal of Cancer 2017, 8(7):1214-1222.

11. Shah N, Parikh V, Patel N, Patel N, Badheka A, Deshmukh A, Rathod A, Lafferty J: Neutrophil lymphocyte ratio significantly improves the Framingham risk score in prediction of coronary heart disease mortality: insights from the National Health and Nutrition Examination Survey-III. International journal of cardiology 2014, 171(3):390-397.

12. Kim S, Eliot M, Koestler DC, Wu WC, Kelsey KT: Association of Neutrophil-to-Lymphocyte Ratio With Mortality and Cardiovascular Disease in the Jackson Heart Study and Modification by the Duffy Antigen Variant. JAMA cardiology 2018, 3(6):455-462.

13. Wang X, Zhang G, Jiang X, Zhu H, Lu Z, Xu L: Neutrophil to lymphocyte ratio in relation to risk of all-cause mortality and cardiovascular events among patients undergoing angiography or cardiac revascularization: a meta-analysis of observational studies. Atherosclerosis 2014, 234(1):206-213.

14. Zhang J, Cai L, Song Y, Shan B, He M, Ren Q, Chen C, Liu Z, Zeng Y, Xu J: Prognostic role of neutrophil lymphocyte ratio in patients with spontaneous intracerebral hemorrhage. Oncotarget 2017, 8(44):77752-77760.

15. Tokgoz S, Keskin S, Kayrak M, Seyithanoglu A, Ogmegul A: Is neutrophil/lymphocyte ratio predict to short-term mortality in acute cerebral infarct independently from infarct volume? Journal of stroke and cerebrovascular diseases: the official journal of National Stroke Association 2014, 23(8):2163-2168.

16. Wang F, Xu F, Quan Y, Wang L, Xia JJ, Jiang TT, Shen LJ, Kang WH, Ding Y, Mei LX et al: Early increase of neutrophilto-lymphocyte ratio predicts 30-day mortality in patients with spontaneous intracerebral hemorrhage. CNS neuroscience \& therapeutics 2019, 25(1):30-35. 
17. Jiang C, Thomas GN, Lam TH, Schooling CM, Zhang W, Lao X, Adab P, Liu B, Leung GM, Cheng KK: Cohort profile: The Guangzhou Biobank Cohort Study, a Guangzhou-Hong Kong-Birmingham collaboration. Int J Epidemio/ 2006, 35(4):844-852.

18. Deng HB, Macfarlane DJ, Thomas GN, Lao XQ, Jiang CQ, Cheng KK, Lam TH: Reliability and validity of the IPAQChinese: the Guangzhou Biobank Cohort study. Medicine and science in sports and exercise 2008, 40(2):303-307.

19. Zhou Z, Lu J, Liu WW, Manaenko A, Hou X, Mei Q, Huang JL, Tang J, Zhang JH, Yao H et al: Advances in stroke pharmacology. Pharmacology \& therapeutics 2018, 191:23-42.

20. Dziedzic T: Systemic inflammation as a therapeutic target in acute ischemic stroke. Expert review of neurotherapeutics 2015, 15(5):523-531.

21. Murray KN, Buggey HF, Denes A, Allan SM: Systemic immune activation shapes stroke outcome. Mol Cell Neurosci 2013, 53:14-25.

22. Qun S, Tang Y, Sun J, Liu Z, Wu J, Zhang J, Guo J, Xu Z, Zhang D, Chen Z et al: Neutrophil-To-Lymphocyte Ratio Predicts 3-Month Outcome of Acute Ischemic Stroke. Neurotox Res 2017, 31(3):444-452.

23. Gistera A, Hansson GK: The immunology of atherosclerosis. Nature reviews Nephrology 2017, 13(6):368-380.

24. Rosales C: Neutrophil: A Cell with Many Roles in Inflammation or Several Cell Types? Frontiers in physiology 2018, 9:113.

25. Quillard T, Franck G, Mawson T, Folco E, Libby P: Mechanisms of erosion of atherosclerotic plaques. Current opinion in lipidology 2017, 28(5):434-441.

26. Valles J, Lago A, Santos MT, Latorre AM, Tembl JI, Salom JB, Nieves C, Moscardo A: Neutrophil extracellular traps are increased in patients with acute ischemic stroke: prognostic significance. Thrombosis and haemostasis 2017, 117(10):1919-1929.

27. Laridan E, Denorme F, Desender L, Francois O, Andersson T, Deckmyn H, Vanhoorelbeke K, De Meyer SF: Neutrophil extracellular traps in ischemic stroke thrombi. Annals of neurology 2017, 82(2):223-232.

28. Kyaw T, Tipping P, Bobik A, Toh BH: Opposing roles of B lymphocyte subsets in atherosclerosis. Autoimmunity 2017, 50(1):52-56.

29. Nam KW, Kwon HM, Jeong HY, Park JH, Kim SH, Jeong SM: High neutrophil to lymphocyte ratios predict intracranial atherosclerosis in a healthy population. Atherosclerosis 2018, 269:117-121.

30. Suh B, Shin DW, Kwon HM, Yun JM, Yang HK, Ahn E, Lee H, Park JH, Cho B: Elevated neutrophil to lymphocyte ratio and ischemic stroke risk in generally healthy adults. PloS one 2017, 12(8):e0183706.

31. Saliba W, Barnett-Griness O, Elias M, Rennert G: Neutrophil to lymphocyte ratio and risk of a first episode of stroke in patients with atrial fibrillation: a cohort study. Journal of thrombosis and haemostasis: JTH2015, 13(11):19711979.

\section{Figures}


30,430 participants recruited for baseline of the

Guangzhou Biobank Cohort Study

1646 participants with incomplete information on blood cell counts, hypertension, diabetes, dyslipidemia, smoking, alcohol drinking, physical activity, BMI, self-rated health, cancer and genitourinary disease or chest disease

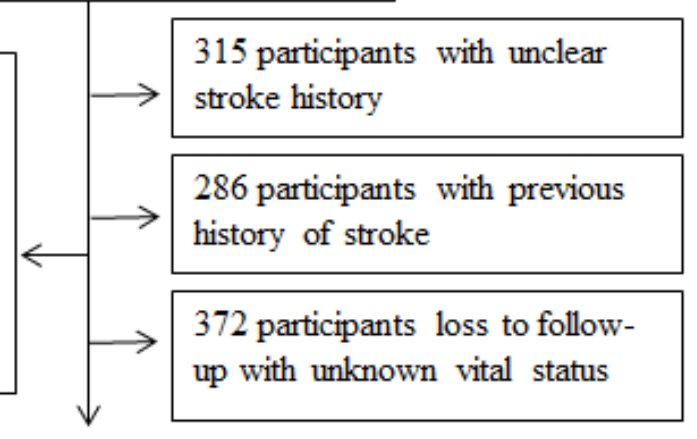

27,811 participants being free of stroke at baseline were recorded in this study (from September 2003 to February 2008)

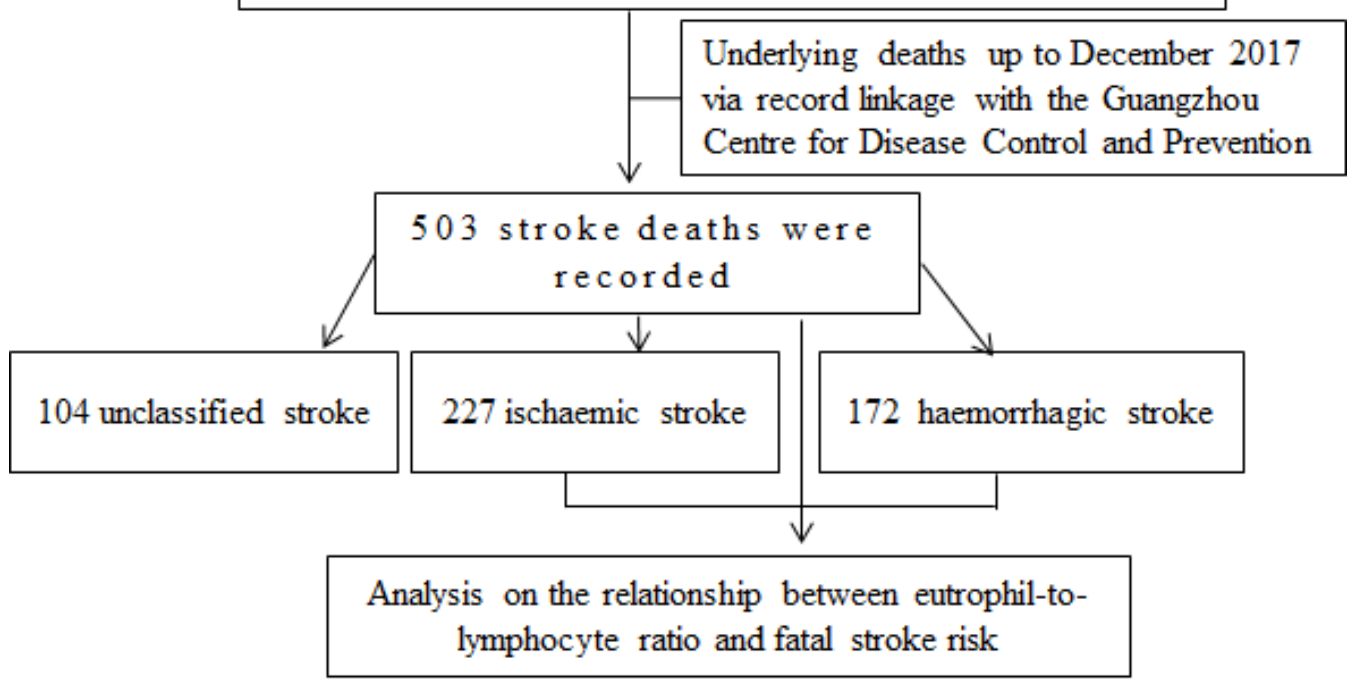

Figure 1

Flow diagram of participants selected for the analysis of this study. 


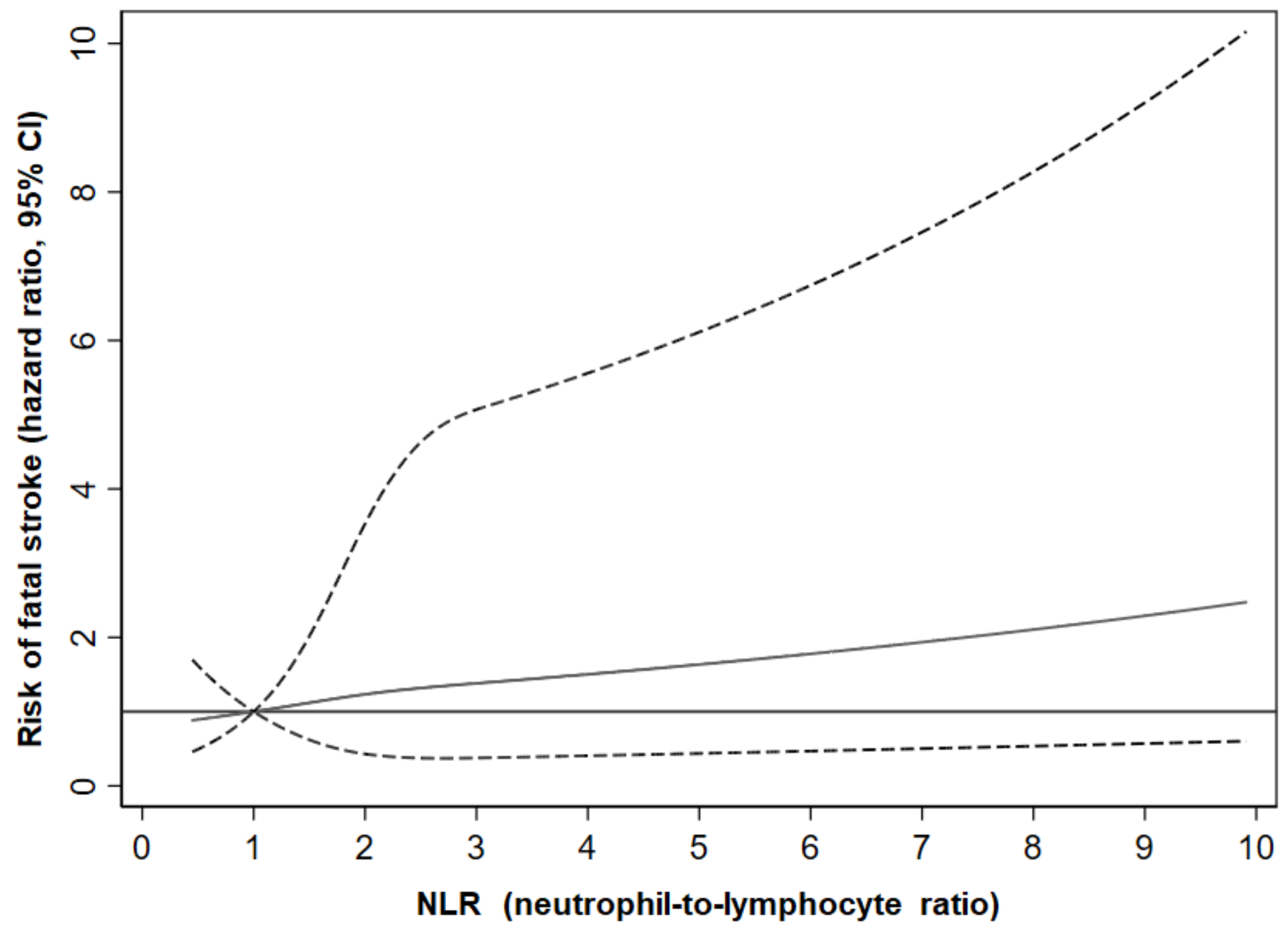

Figure 2

Association between the NLR and the risk of fatal stroke with the RCS model. The solid blue line is the multivariable adjusted hazard ratio, with dashed lines showing 95\% confidence intervals with three knots. A multivariate model adjusted for sex, age, education, occupation, diabetes, hypertension, dyslipidaemia, smoking, alcohol consumption, physical activity, body mass index, self-rated health, cancer, genitourinary diseases, chest disease and platelet count was used. 
A

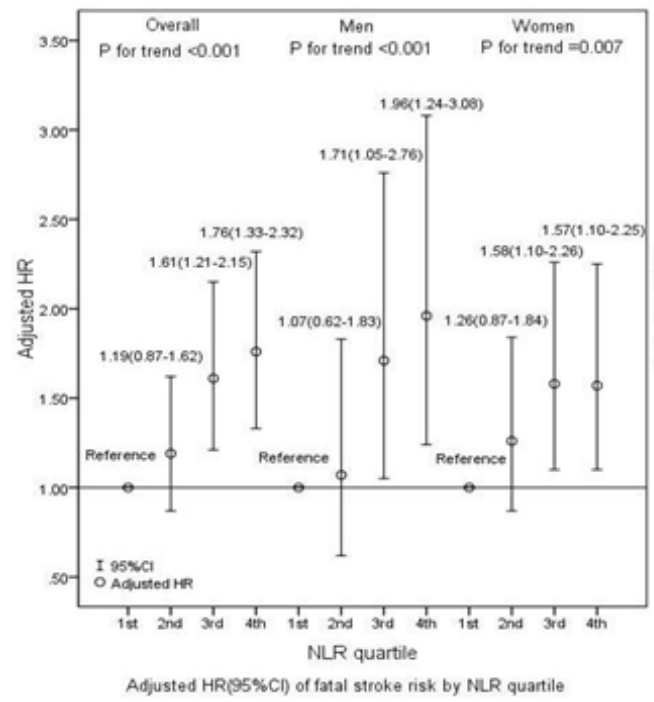

B

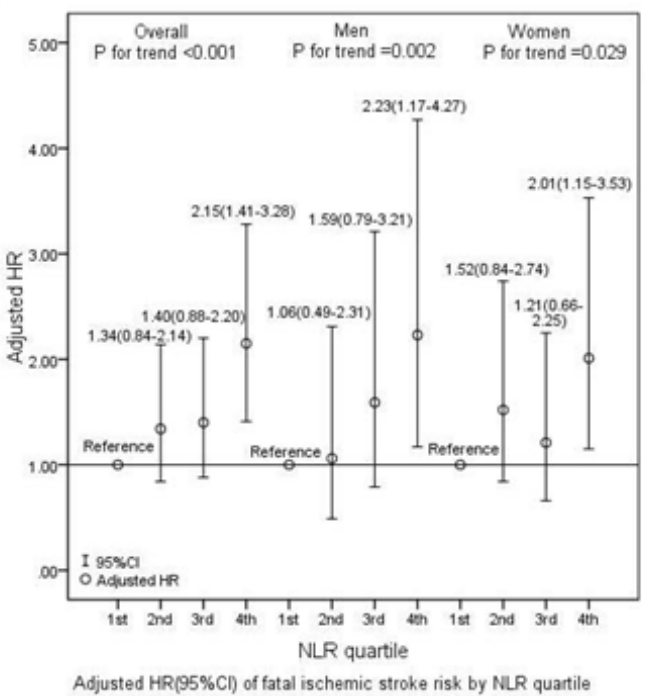

C

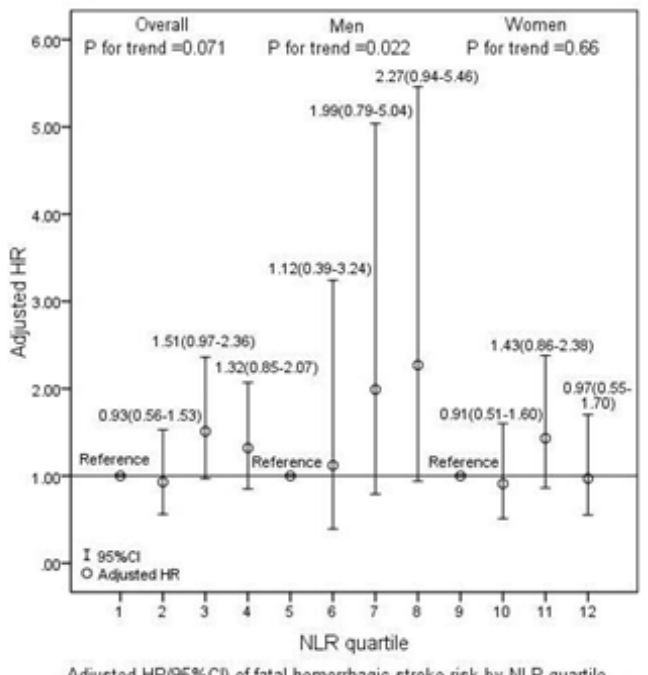

Adjusted HR(95\%CI) of fatal hemorrhagic stroke risk by NLR quartile

\section{Figure 3}

Association between the NLRs and the risk of fatal stroke in participants of the GBCS. A, B and C plots an adjusted hazard ratio and $95 \%$ confidence interval for neutrophil-to-lymphocyte ratio' quartile alongside with the $\mathrm{P}$ value for trend. The adjusted confounders include age, sex, hypertension, diabetes, dyslipidemia, smoking, alcohol drinking, physical activity, body mass index, self-rated health, cancer, genitourinary disease, chest disease and platelet count. 